PROCEEDINGS OF THE

AMERICAN MATHEMATICAL SOCIETY

Volume 125, Number 1, January 1997, Pages 255-258

S 0002-9939(97)03721-0

\title{
A GENERALIZATION OF THE CLASSICAL SPHERE THEOREM
}

\author{
CHANGYU XIA
}

(Communicated by Christopher Croke)

\begin{abstract}
In this paper, we prove a sphere theorem for Riemannian manifolds with partially positive curvature which generalizes the classical sphere theorem.
\end{abstract}

\section{INTRODUCTION}

The celebrated Rauch-Berger-Klingenberg sphere theorem [1] states that, if $M$ is an $n$-dimensional complete simply connected Riemannian manifold with sectional curvature $K_{M}$ satisfying $1<K_{M} \leq 4$, then $M$ is homeomorphic to an $n$-sphere $S^{n}$. In 1977, Grove and Shiohama [2] generalized this sphere theorem to the following form : If the sectional curvature and diameter of $M$ satisfy $d(M)>\frac{\pi}{2}$ and $K_{M} \geq 1$ then $M$ is homeomorphic to $S^{n}$. In this paper, we shall prove a sphere theorem for manifolds with partially positive curvature which also generalizes the RauchBerger-Klingenberg sphere theorem.

Before stating our results, we fix some notations for the $k$-th Ricci curvature of a Riemannian manifold (Cf. [4], [6]). Let $M$ be an $n$-dimensional Riemannian manifold. If, for any point $x \in M$ and any $(k+1)$-mutually orthogonal unit tangent vectors $e, e_{1}, \ldots, e_{k} \in T_{x} M$, we have $\sum_{i=1}^{k} K\left(e \wedge e_{i}\right) \geq k c$ (resp., $\left.>k c\right)$, we say that the $k$-th Ricci curvature of $M$ is not less than $k c$ ( resp., larger than $k c)$ and denote this fact by $\operatorname{Ric}_{(k)}(M) \geq k c$ (resp., $\left.>k c\right)$. Here, $K\left(e \wedge e_{i}\right)$ denotes the sectional curvature of the plane spanned by $e$ and $e_{i}(1 \leq i \leq k)$. Thus, $\operatorname{Ric}_{(1)}(M) \geq c$ (resp., $>c$ ) is equivalent to $K_{M} \geq c$ (resp., $>c$ ) and $\operatorname{Ric}_{(n-1)}(M) \geq(n-1) c$ (resp., $\left.>(n-1) c\right)$ is equivalent to the fact that the Ricci curvature of $M$ satisfies $\operatorname{Ric}_{M} \geq(n-1) c$ (resp., $\left.>(n-1) c\right)$. Also, it is easy to see that $\operatorname{Ric}_{(k)}(M) \geq k c$ (resp., $\left.>k c\right)$ implies $\operatorname{Ric}_{(m)}(M) \geq m c$ (resp., $>m c$ ) for $m \geq k$

Now we can state our main theorem as follows.

Theorem 1. Let $M$ be an $n(\geq 3)$-dimensinal complete simply connected Riemannian manifold with $\operatorname{Ric}_{\left(\left[\frac{n+1}{2}\right]\right)}(M)>\left[\frac{n+1}{2}\right]$. If there exists a point $p \in M$ such that the injectivity radius $i_{p}$ of $M$ at $p$ satisfies $i_{p} \geq \frac{\pi}{2}$, then $M$ is homeomorphic to an $n$-sphere $S^{n}$.

Received by the editors August 2, 1995.

1991 Mathematics Subject Classification. Primary 53C20.

Key words and phrases. Curvature, sphere theorem.

This work was partially supported by the JSPS postdoctoral fellowship and National Natural Science Foundation of China.

(C)1997 American Mathematical Society 
Let $M=C P^{2}(4)$ be the complex projective space of complex dimension 2 and of holomorphic sectional curvature 4 . Then the injectivity radius of $M$ is $\frac{\pi}{2}$ and $\operatorname{Ric}_{(3)}(M)=6>3$, but $M$ is not homeomorphic to $S^{4}$. This example shows that the curvature condition " $\operatorname{Ric}_{\left(\left[\frac{n+1}{2}\right]\right)}(M)>\left[\frac{n+1}{2}\right]$ " in Theorem 1 can't be weakened to " $\operatorname{Ric}_{\left(1+\left[\frac{n+1}{2}\right]\right)}(M)>1+\left[\frac{n+1}{2}\right]$ ".

We remark that when $n=3$, Theorem 1 is a special case of Hamilton's theorem [3]. An important result of Hartman [4] states that if $M$ is an $n(\geq 3)$-dimensional complete simply connected Riemannian manifold with $K_{M} \leq 4$ and $\operatorname{Ric}_{(n-2)}(M) \geq$ $n-2$, then the injectivity radius of $M$ satisfies $i(M) \geq \frac{\pi}{2}$. Thus, Theorem 1 immediately implies the following generalized form of the classical sphere theorem:

Theorem 2. Let $M$ be an $n(\geq 3)$-dimensional complete simply connected Riemannian manifold. If $K_{M} \leq 4$ and $\operatorname{Ric}_{\left(\left[\frac{n+1}{2}\right]\right)}(M)>\left[\frac{n+1}{2}\right]$, then $M$ is homeomorphic to $S^{n}$.

\section{A Proof of Theorem 1}

Before proving Theorem 1, we list some basic facts we need. Let $M$ be a compact $n$-dimensional Riemannian manifold with boundary. For any $1 \leq k \leq n-1$, let $\Lambda_{k}: \partial M \rightarrow R$ be the function on $\partial M$ defined by $\Lambda_{k}(p)=$ the minimum of all sums of any $k$ eigenvalues of the second fundamental form of $\partial M$ at $p$ with respect to the outward-pointing unit normal $N$ at $p$. $\partial M$ is called $k$-convex if $\Lambda_{k}>0$ on $\partial M$.

Lemma 1 ([6]). Let $M$ be an n-dimensional compact Riemannian manifold with boundary $\partial M$ and let $k$ be an integer satisfying $1 \leq k \leq n-1$. If $\operatorname{Ric}_{(k)}(M)>0$ and $M$ has $k$-convex boundary, then $M$ has the homotopy type of a $C W$ complex obtained from $\partial M$ by attaching a finite number of cells each of dimension $\geq n-k+1$.

Proof of Theorem 1. When $n=3$, Theorem 1 follows from Hamilton's theorem [3]. Thus, we can assume $n \geq 4$. Let $B(p, r)$ be the open geodesic ball of radius $r$ with center $p$. Since the injectivity radius of $M$ at $p$ is not less than $\frac{\pi}{2}$, thus, for any $r \in\left(0, \frac{\pi}{2}\right), B(p, r)$ is homeomorphic to an $n$-disk and the boundary $\partial B(p, r)$ of $B(p, r)$ is a smooth hypersurface and it is homeomorphic to an $(n-1)$-sphere $S^{n-1}$. From $\operatorname{Ric}_{\left(\left[\frac{n+1}{2}\right]\right)}(M)>\left[\frac{n+1}{2}\right]$, we know that $\operatorname{Ric}_{M}>n-1$, thus $M$ is compact by Bonnet-Myers theorem [1], and so we can find a positive constant $\delta$ such that $\operatorname{Ric}_{\left(\left[\frac{n+1}{2}\right]\right)}(M)>\left[\frac{n+1}{2}\right]+\delta$. Take $l=\frac{\pi}{2 \sqrt{1+\frac{\delta}{\left[\frac{n+1}{2}\right]}}}$; then $l<\frac{\pi}{2}$. Now, we

want to show that $M-B(p, l)$ has $\left[\frac{n+1}{2}\right]$-convex boundary. To do this, we denote by $\nabla$ the Riemannian connection of $M-B(p, l)$ and $S$ the second fundamental form of $M-B(p, l)$ with respect to the outward-pointing unit normal $N$, i.e., $S(u, v)=\left\langle\nabla_{u} N, v\right\rangle$ for $u, v \in T \partial B(p, l)$. Let $q$ be any point on the boundary of $M-B(p, l)$ and $\gamma:[0, l] \rightarrow \bar{B}(p, l)$ be a normal geodesic from $p$ to $q$ that realizes the minimum distance between them. An argument of the first variation of arc-length shows that $\gamma$ strikes $\partial B(p, l)$ orthogonally. For any $k$ orthonormal vectors $e_{1}, \ldots, e_{k}$ in $T_{q} \partial B(p, l)$, we denote by $E_{i}(s)$ the parallel translate of $e_{i}$ to $\gamma(s)$ along $\gamma$ and define $W_{i}(s)=\sin \frac{\pi s}{2 l} E_{i}(s), i=1, \ldots, k$. Each vector field $W_{i}$ gives rise to a variation with length $L_{W_{i}}(t)(t \in(-\epsilon, \epsilon))$ of the variational curves of the geodesic $\gamma$ keeping one end point $p$ fixed and other end points on $\partial B(p, l)$. The first variation of the arc-length $L_{W_{i}}^{\prime}(0)=0$, and the second variation of the arc-length $L_{W_{i}}^{\prime \prime}(0) \geq 0, i=1, \ldots, k$, since $\gamma$ is a minimizing geodesic from $p$ to $q$. It 
then follows from the second variation formula of arc-length ( see p.99 in [5]) that for $i=1, \ldots, k$,

$$
\begin{aligned}
0 \leq & L_{W_{i}}^{\prime \prime}(0) \\
= & \left\langle\nabla_{e_{i}} e_{i}, \gamma^{\prime}(l)\right\rangle+\int_{0}^{l}\left\{\left|\nabla_{\gamma^{\prime}(s)} W_{i}(s)\right|^{2}\right. \\
& \left.-\left\langle R\left(\gamma^{\prime}(s), W_{i}(s)\right) W_{i}(s), \gamma^{\prime}(s)\right\rangle\right\} d s \\
= & S\left(e_{i}, e_{i}\right)+\int_{0}^{l}\left\{\left|\nabla_{\gamma^{\prime}(s)} W_{i}(s)\right|^{2}\right. \\
& \left.-\left\langle R\left(\gamma^{\prime}(s), W_{i}(s)\right) W_{i}(s), \gamma^{\prime}(s)\right\rangle\right\} d s,
\end{aligned}
$$

where $R$ is the curvature tensor of $M$.

Thus, from $\operatorname{Ric}_{\left(\left[\frac{n+1}{2}\right]\right)}>\left[\frac{n+1}{2}\right]+\delta$ and (1), we get

$$
\begin{aligned}
\sum_{i=1}^{\left[\frac{n+1}{2}\right]} S\left(e_{i}, e_{i}\right) \geq & -\sum_{i=1}^{\left[\frac{n+1}{2}\right]} \int_{0}^{l}\left\{\left|\nabla_{\gamma^{\prime}(s)} W_{i}(s)\right|^{2}\right. \\
& \left.-\left\langle R\left(\gamma^{\prime}(s), W_{i}(s)\right) W_{i}(s), \gamma^{\prime}(s)\right\rangle\right\} d s \\
= & -\sum_{i=1}^{\left[\frac{n+1}{2}\right]} \int_{0}^{l}\left\{\frac{\pi^{2}}{4 l^{2}} \cos ^{2} \frac{\pi s}{2 l}\right. \\
& \left.-\sin ^{2} \frac{\pi s}{2 l}\left\langle R\left(\gamma^{\prime}(s), E_{i}(s)\right) E_{i}(s), \gamma^{\prime}(s)\right\rangle\right\} d s \\
> & -\left[\frac{n+1}{2}\right] \int_{0}^{l}\left(\frac{\pi^{2}}{4 l^{2}} \cos ^{2} \frac{\pi s}{2 l}-\left(1+\frac{\delta}{\left[\frac{n+1}{2}\right]}\right) \sin ^{2} \frac{\pi s}{2 l}\right) d s \\
= & -\left[\frac{n+1}{2}\right] \cdot \frac{2 l}{\pi} \int_{0}^{\frac{\pi}{2}}\left(\frac{\pi^{2}}{4 l^{2}} \cos ^{2} s-\left(1+\frac{\delta}{\left[\frac{n+1}{2}\right]}\right) \sin ^{2} s\right) d s \\
= & -\left[\frac{n+1}{2}\right] \cdot \frac{l}{2}\left(\frac{\pi^{2}}{4 l^{2}}-\left(1+\frac{\delta}{\left[\frac{n+1}{2}\right]}\right)\right) \\
= & 0 .
\end{aligned}
$$

Therefore, $M-B(p, l)$ has $\left[\frac{n+1}{2}\right]=n-\left[\frac{n}{2}\right]$-convex boundary. From Lemma 1 we conclude that $M-B(p, l)$ has the homotopy type of a $C W$ complex obtained from $\partial B(p, l) \simeq S^{n-1}$ by attaching a finite number of cells each of dimension $\geq\left[\frac{n}{2}\right]+1$. So does $M$ since $M=B(p, l) \cup(M-B(p, l))$ and $B(p, l) \simeq D^{n}$, the $n$-disk. Thus, $\pi_{i}(M, B(p, l))=0$ for $1 \leq i \leq\left[\frac{n}{2}\right]$. From the homotopy exact sequence we know that $\pi_{i}(M)=0$ for $1 \leq i \leq\left[\frac{n}{2}\right]$. By a basic argument in topology, one can deduce

that $M$ is homeomorphic to the sphere $S^{n}$. This completes the proof of Theorem 1.

\section{REFERENCES}

[1] J. Cheeger and D. Ebin, Comparison Theorems in Riemannian Geometry, North-holland, New-York, 1975.

[2] K. Grove and K. Shiohama, A generalized sphere theorem, Ann. of Math., 106 (1977), 201211. MR 58:18268 
[3] R. Hamilton, Three manifolds with positive Ricci curvature, J. Differential Geom., 17 (1982), 255-306. MR 84a:53050

[4] P. Hartman, Oscillation criteria for self-adjoint second-order differential systems and " principal sectional curvature " , J. Differential Equations 34 (1979), 326-338. MR 81a:34034

[5] W. Klingenberg, Riemannian Geometry, Berlin, New York : de Gruyter, 1982. MR 84j:53001

[6] $\mathrm{H}$. Wu, Manifolds of partially positive curvature, Indiana Univ. Math. J., 36 (1987), 525-548. MR 88k:53068

[7] C.Y. Xia, Rigidity and sphere theorem for manifolds with positive Ricci curvature, manuscripta math., 85 (1994), 79-87. MR 95j:53057

Department of Mathematics, University of Science and Technology of China, Hefei, Anhui 230026, People's Republic of China

Current address: Instituto de Matemática Pure e Aplicada, Estrada Dona Castorina, 110, 22460-320 Rio de Janeiro RJ, Brasil

E-mail address: xiacy@impa.br 